$$
\begin{gathered}
\text { 상향링크 셀룰러 시스템에서 채널 상관 기반의 } \\
\text { 협력 사용자 스케줄링 기법 } \\
\text { 오동찬 }{ }^{\circ}, \text { 이진우, 이용환 } \\
\text { 서울대학교 전기컴퓨터 공학부 }
\end{gathered}
$$

\title{
Correlation-Based Cooperative User Scheduling in the Uplink of Cellular Systems
}

\author{
Dong-Chan $\mathrm{Oh}^{\mathrm{O}}$, Jin-Woo Lee, and Yong-Hwan Lee \\ School of Electrical Engineering and INMC Seoul National University \\ Kwanak P. O. Box 34, Seoul 151-600 Korea \\ \{mac81, jinu\}@ttl.snu.ac.kr, ylee@snu.ac.kr
}

\begin{abstract}
In this paper, we consider multi-user scheduling to avoid other cell interference (OCI) in the uplink of cellular systems. The base station (BS) determines a user group that can minimize the interference from other cells by exploiting the spatial correlation matrix of users from adjacent BSs. The proposed scheme is applicable to multi-input multi-output (MIMO) as well as single-input multi-output (SIMO) environments by applying an eigen-beamforming technique, enabling the use of flexible antenna structures at the transmitter. Simulation results show that the proposed multi-cell scheduling significantly increase the ergodic capacity by avoiding the OCI compared to conventional scheduling schemes, particularly in high mobility and highly correlated channel environments.
\end{abstract}

\section{Introduction}

Increasing demand for packet data applications in the uplink of cellular networks has encouraged the reuse of frequency spectrum to increase the system capacity. However, when the frequency resource is universally reused, the uplink capacity can severely be degraded by interference from users in the adjacent cells, so-called other-cell interference (OCI) [1]-[3]. Since the received signal power transmitted near the cell boundary is much weaker than that near the cell center, the OCI can adversely affect the uplink signal transmitted near the cell boundary. It is desirable to mitigate the OCI to increase the throughput of the uplink signal transmitted near the cell boundary.

Assume that base stations (BSs) near the target BS are fully coordinated (i.e., fully share instantaneous channel state information (CSI) of all users near the cell boundary with no delay). Then, each BS can schedule users to minimize the OCI effect, improving the uplink throughput near the cell boundary [2]. In practice, however, it takes time to exchange the CSI (e.g., the mobile WiMAX and 3GPP LTE set a constraint on the maximum cooperative interval in an order of ten seconds to minutes [4]). As a consequence, the use of a full coordinated scheduling scheme may not be feasible in practice.

Instead of the coordination, the use of a Max-SNR scheduling policy has been considered based on instantaneous CSI [5]. However, it can suffer from socalled channel mismatch problem, mainly due to the time delay between the time of channel estimation and signal reception at the BS. This channel mismatch problem makes the reception performance worse as the user mobility increases [6]. To alleviate this problem, the use of longterm CSI (e.g., spatial correlation) has been considered [3],[7],[8], which varies slowly. However, most of spatial correlation-based scheduling schemes have been proposed in single-cell environments. Although they can somewhat alleviate the channel mismatch problem, they still suffer from the OCI effect when applied to multi-cell environments.

In this paper, we consider cooperative multi-user scheduling based on the spatial correlation to maximize the ergodic capacity of the uplink in multi-cell environments. The BS can schedule users near the cell boundary based on

This research was supported by Seoul R\&BD Program (10544). 


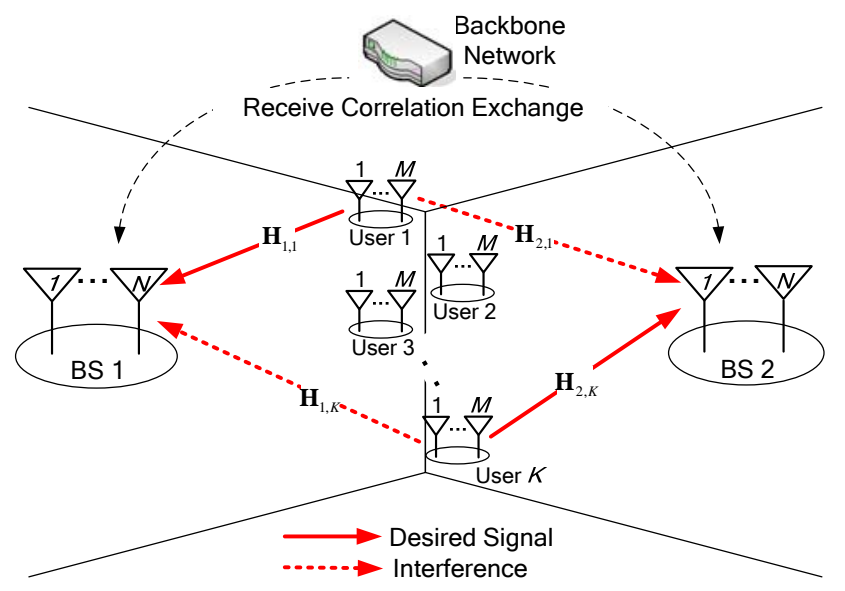

Fig. 1. Modeling of a multi-user MIMO uplink system

the transmit/receive correlation matrix. Since the spatial correlation varies slowly, it can be exchanged among the BSs through a backbone network without serious outdated problem [6]. Based on the spatial correlation matrix of users near the cell boundary, each BS selects its users minimally affected by the OCI from adjacent cells, maximizing the ergodic capacity of the uplink. Since the proposed scheme makes the use of long-term CSI, it can be quite effective in high mobility and highly correlated channel environments.

The rest of the paper is organized as follows. Section II describes the system model in consideration. Section III proposes a cooperative user scheduling scheme that minimize the OCI effect on the uplink signal by exploiting the spatial correlation of users. Section IV verifies the proposed scheduling scheme by computer simulation. Finally, conclusions are given in Section V.

\section{Multi-user MIMO Uplink Channel}

Consider the uplink of a multi-input and multi-output (MIMO) cellular system comprising $B$ BSs, where the BS has $N$ receive antennas and $K$ users have $M$ transmit antennas. Fig. 1 illustrates a MIMO cellular system where two BSs are serving $K$ users near the cell boundary. Let $s_{k}$ be the transmit signal from user $k$, $P_{i, k}$ be the average signal power transmitted from user $k$ to BS $i, \mathbf{u}_{k}$ be the transmit beam weight vector of user $k$, and $\mathbf{H}_{i, k}$ be the $(N \times M)$ channel matrix between user $k$ and BS $i$. Then, the received signal at BS $i$ can be represented as

$$
\mathbf{y}_{i}=\sum_{k=1}^{K} \mathbf{H}_{i, k} \mathbf{u}_{k} s_{k}+\mathbf{n}_{i}
$$

where $\mathbf{n}_{i}$ is the $(N \times 1)$ additive white Gaussian noise (AWGN) vector with covariance $N_{0} \mathbf{I}_{N}$.

Assuming that the channels are spatially correlated, $\mathbf{H}_{i, k}$ can be represented as [9]

$$
\mathbf{H}_{i, k}=\mathbf{R}_{i, k}^{1 / 2} \tilde{\mathbf{H}}_{i, k} \mathbf{T}_{i, k}^{1 / 2}
$$

where $\tilde{\mathbf{H}}_{i, k}$ is an $(N \times M)$ matrix whose entries are independent and identically distributed (i.i.d) zero-mean complex Gaussian with unit variance, and $\mathbf{R}_{i, k}$ and $\mathbf{T}_{i, k}$ are $(N \times N)$ receive and $(M \times M)$ transmit correlation matrix from user $k$ to BS $i$, respectively. $\mathbf{H}_{i, k}$ can also be represented as

$$
\begin{aligned}
\mathbf{H}_{i, k} & =\left[\mathbf{h}_{i, k}^{(1)}, \mathbf{h}_{i, k}^{(2)}, \cdots, \mathbf{h}_{i, k}^{(M)}\right] \\
& =\left[\mathbf{g}_{i, k}^{(1)^{*}}, \mathbf{g}_{i, k}^{(2)^{*}}, \cdots, \mathbf{g}_{i, k}^{(N)^{*}}\right]^{*}
\end{aligned}
$$

where $\mathbf{h}_{i, k}^{(l)}, \quad l=1,2, \cdots, M$, is the $(N \times 1)$ receive channel vector between the BS and the $l$-th transmit antenna of user $k, \mathbf{g}_{i, k}^{(m)}, \quad m=1,2, \cdots, N$, is $(1 \times M)$ transmit channel vector between user $k$ and the $m$-th receive antenna of the $B S$, and the superscript * denotes conjugate transpose.

The receive correlation matrix $\mathbf{R}_{i, k}$ is equal to the covariance of $\mathbf{h}_{i, k}^{(l)}$ when excited by any transmit antenna $l=1,2, \cdots, M$, and is the same for all transmit antennas [9]. Simply, it can be represented in an exponential decay form [10]

$$
\begin{aligned}
\mathbf{R}_{i, k} & =E\left\{\mathbf{h}_{i, k}^{(l)} \mathbf{h}_{i, k}^{(l) *}\right\} \\
& =\left[\begin{array}{cccc}
1 & \rho_{i, k} & \cdots & \rho_{i, k}^{N} \\
\rho_{i, k}^{*} & 1 & & \rho_{i, k}^{(N-1)} \\
\vdots & & \ddots & \vdots \\
\rho_{i, k}^{N^{*}} & \rho_{i, k}^{(N-1)^{*}} & \cdots & 1
\end{array}\right]
\end{aligned}
$$

where $\rho_{i, k}=\left|\rho_{i, k}\right| e^{j \theta_{i, k}} \quad$ is the receive correlation coefficient between the adjacent receive antennas of BS $i$ associated with any transmit antenna of user $k$. Here $\left|\rho_{i, k}\right| \quad\left(0 \leq\left|\rho_{i, k}\right|<1\right)$ and $\theta_{i, k} \quad\left(-\pi<\theta_{i, k} \leq \pi\right) \quad$ denote the magnitude and the phase of the receive correlation, respectively.

This model can occur when the receive antennas receive scatters with the same angle spectra. This condition arises if the receive antennas are closely located and have the same radiation pattern. In a similar manner, the transmit antenna correlation matrix $\mathbf{T}_{i, k}$ defined by the covariance of $\mathbf{g}_{i, k}^{(m)}, \quad m=1,2, \cdots, N$, can be represented as [10]

$$
\begin{aligned}
\mathbf{T}_{i, k} & =E\left\{\mathbf{g}_{i, k}^{(m)^{*}} \mathbf{g}_{i, k}^{(m)}\right\} \\
& =\left[\begin{array}{cccc}
1 & \mu_{i, k} & \cdots & \mu_{i, k}^{M} \\
\mu_{i, k}^{*} & 1 & & \mu_{i, k}^{(M-1)} \\
\vdots & & \ddots & \vdots \\
\mu_{i, k}^{M^{*}} & \mu_{i, k}^{(M-1)^{*}} & \cdots & 1
\end{array}\right]
\end{aligned}
$$

where $\mu_{i, k}=\left|\mu_{i, k}\right| e^{j \phi_{i, k}} \quad$ is the transmit correlation coefficient between the adjacent transmit antennas of user $k$ associated with any receive antenna of BS $i$. Here $\left|\mu_{i, k}\right| \quad\left(0 \leq\left|\mu_{i, k}\right|<1\right) \quad$ and $\phi_{i, k} \quad\left(-\pi<\phi_{i, k} \leq \pi\right) \quad$ denote the magnitude and the phase of the transmit correlation, respectively. 


\section{Proposed Multi-cell Cooperative Scheduling Scheme}

Assume that each user transmits the signal with a beam weight using $M$ transmit antennas and the BS receives it using $N$ receive antennas. The singular value decomposition (SVD) technique was previously applied to the uplink of a multi-user MIMO system [12], where the signal is transmitted with a beam weight generated by the right singular vector corresponding to the largest singular value of the instantaneous CSI. However, the use of this beam weight may suffer from the channel mismatch problem. Moreover, since this scheme requires instantaneous CSI, causing a large feedback signaling overhead.

To alleviate this problem, we consider the use of an eigen-value decomposition (EVD) technique for the generation of transmit beam weight. Since the transmit correlation matrix $\mathbf{T}_{i, k}$ is Hermitian, it can be represented as

$$
\mathbf{T}_{i, k}=\mathbf{V}_{i, k} \sum_{i, k} \mathbf{V}_{i, k}^{*}
$$

where $\sum_{i, k}$ is a diagonal matrix whose diagonal elements $\lambda_{i, k}^{(1)}>\lambda_{i, k}^{(2)}>\cdots>\lambda_{i, k}^{(M)}$ are the eigen-values and $\mathbf{V}_{i, k}$ is an unitary matrix whose columns $\left[\mathbf{v}_{i, k}^{(1)}, \mathbf{v}_{i, k}^{(2)}, \cdots, \mathbf{v}_{i, k}^{(M)}\right]$ are the eigen-vectors corresponding to the eigen-values. Obviously, $\mathbf{v}_{i, k}^{(1)}$ and $\mathbf{v}_{i, k}^{(M)}$ are the eigen-beam weight maximizing and minimizing the average SNR, respectively. It is known that the use of an eigen-beam weight corresponding to the largest eigen-value of the channel covariance matrix is asymptotically optimum when the spatial correlation is high [13]. Since it is of concern on correlated channel environments, we consider the use of eigen-beamforming that allocates all power to the strongest eigen-dimension of the channel.

For ease of description, assume that BS 1 is the target BS. Letting $Q(i)$ be the index of a user scheduled by BS $i$, the received signal at BS 1 can be represented as

$$
\begin{aligned}
\mathbf{y}_{1}= & \mathbf{H}_{1, Q(1)} \mathbf{u}_{Q(1)} S_{Q(1)}+\sum_{i=2}^{B} \mathbf{H}_{1, Q(i)} \mathbf{u}_{Q(i)} S_{Q(i)}+\mathbf{n}_{1} \\
= & \mathbf{H}_{1, Q(1)} \mathbf{v}_{1, Q(1)}^{(1)} S_{Q(1)}+\sum_{i=2}^{B} \mathbf{H}_{1, Q(i)} \mathbf{v}_{i, Q(i)}^{(1)} S_{Q(i)}+\mathbf{n}_{1} \\
= & \sqrt{\lambda_{1, Q(1)}^{(1)}} \mathbf{R}_{1, Q(1)}^{1 / 2} \tilde{\mathbf{h}}_{1, Q(1)} S_{Q(1)} \\
& \quad+\sum_{i=2}^{B} \mathbf{R}_{1, Q(i)}^{1 / 2} \tilde{\mathbf{H}}_{1, Q(i)} \mathbf{T}_{1, Q(i)}^{1 / 2} \mathbf{v}_{i, Q(i)}^{(1)} S_{Q(i)}+\mathbf{n}_{1}
\end{aligned}
$$

where the first term is the desired signal from user $Q(1)$, the second term is the OCI from users scheduled by adjacent BSs and the third term is the AWGN vector. Here, $\tilde{\mathbf{h}}$ is an $(N \times 1)$ vector whose entries are independent and identically distributed (i.i.d) zero-mean complex Gaussian random variables with unit variance.

The OCI term defined by $\mathbf{O}_{1}$ can be rewritten as

$$
\begin{aligned}
\mathbf{O}_{1} & \triangleq \sum_{i=2}^{B} \mathbf{R}_{1, Q(i)}^{1 / 2} \tilde{\mathbf{H}}_{1, Q(i)} \mathbf{T}_{1, Q(i)}^{1 / 2} \mathbf{v}_{i, Q(i)}^{(1)} S_{Q(i)} \\
& =\sum_{i=2}^{B} \mathbf{R}_{1, Q(i)}^{1 / 2} \tilde{\mathbf{H}}_{1, Q(i)} \mathbf{V}_{1, Q(i)} \sum_{1, Q(1)}^{1 / 2} \mathbf{V}_{1, Q(i)}^{*} \mathbf{v}_{i, Q(i)}^{(1)} S_{Q(i)} \\
& =\sum_{i=2}^{B} \mathbf{R}_{1, Q(i)}^{1 / 2} \tilde{\mathbf{H}}_{1, Q(i)} \sum_{j=1}^{M} \mathbf{v}_{1, Q(i)}^{(j)}\left(\sqrt{\lambda_{1, Q(i)}^{(j)}} \mathbf{v}_{1, Q(i)}^{(j)^{*}} \mathbf{v}_{i, Q(i)}^{(1)}\right) S_{Q(i)} \\
& =\sum_{i=2}^{B} \mathbf{R}_{1, Q(i)}^{1 / 2} \tilde{\mathbf{h}}_{1, Q(i)}\left(\sum_{j=1}^{M} \sqrt{\lambda_{1, Q(i)}^{(j)}} \delta_{Q(i)}^{(j)}\right) S_{Q(i)}
\end{aligned}
$$

where $\delta_{Q(i)}^{(j)} \triangleq \mathbf{v}_{1, Q(i)}^{(j)^{*}} \mathbf{v}_{i, Q(i)}^{(1)}, j=1,2, \cdots, M$. It can be seen that the OCI power increases as $\delta_{Q(i)}^{(1)}$ increases, while decreasing as $\delta_{Q(i)}^{(M)}$ increases. This implies that the OCI can be minimized in an average sense by making the beam weight of users in cell $i(\neq 1)$ has the same direction as the eigen-vector corresponding to the smallest eigen-value of $\mathbf{T}_{1, Q(i)}$ (i.e., $\mathbf{u}_{Q(i)}=\mathbf{v}_{i, Q_{(i)}^{(1)}}=\mathbf{v}_{1, Q(l)}^{(M)}$ ). On the other hands, the OCI can be maximized in an average sense by making the direction of $\mathbf{u}_{Q(i)}$ and the eigen-vector corresponding to the largest eigen-value of $\mathbf{T}_{1, Q(i)}$ the same.

With the use of the eigen-beamforming, (7) can be rewritten as

$$
\begin{aligned}
\mathbf{y}_{1}= & \sqrt{\lambda_{1, Q(1)}^{(1)}} \mathbf{R}_{1, Q(1)}^{1 / 2} \tilde{\mathbf{h}}_{1, Q(1)} s_{Q(1)} \\
& +\sum_{i=2}^{B} \mathbf{R}_{1, Q(i)}^{1 / 2} \tilde{\mathbf{h}}_{1, Q(i)}\left(\sum_{j=1}^{M} \sqrt{\lambda_{1, Q(i)}^{(j)}} \delta_{Q(i)}^{(j)}\right) s_{Q(i)}+\mathbf{n}_{1} \\
= & \sqrt{\lambda_{1, Q(1)}^{(1)}} \mathbf{h}_{1, Q(1)} S_{Q(1)}+\mathbf{z}_{1}
\end{aligned}
$$

where

$$
\left.\mathbf{z}_{1} \triangleq \sum_{i=2}^{\sqrt{\eta_{1, Q(i)}}}=\sum_{1, Q(i)}^{M} \sqrt{\eta_{1, Q(i)} s_{Q(i)}+\mathbf{n}}\right)^{(j)} \delta_{Q(i)}^{(j)} \text { is an additive }
$$
colored noise term with invertible covariance matrix

$$
\begin{aligned}
\mathbf{K}_{z_{1}} & =E\left\{\mathbf{z}_{1} \mathbf{z}_{1}^{*}\right\} \\
& =N_{0} \mathbf{I}_{N}+\sum_{i=2}^{B} \eta_{1, Q(i)} P_{1, Q(i)} \mathbf{R}_{1, Q(i)} .
\end{aligned}
$$

Assume that the BS determines the receive weight to maximize the output signal-to-interference plus noise ratio (SINR). Then, the optimum received weight can be determined as [11]

$$
\begin{aligned}
\mathbf{w}_{1} & =\mathbf{K}_{z_{1}}^{-1} \mathbf{h}_{1, Q(1)} \\
& =\left(N_{0} \mathbf{I}_{N}+\sum_{i=2}^{B} \eta_{1, Q(i)} P_{1, Q(i)} \mathbf{R}_{1, Q(i)}\right)^{-1} \mathbf{R}_{1, Q(1)}^{1 / 2} \tilde{\mathbf{h}}_{1, Q(1)}
\end{aligned}
$$

The use of receive weight $\mathbf{w}_{1}$ yields an output SINR of user $Q(1)$ represented as

$$
\gamma_{1}=\lambda_{1, Q(1)}^{(1)} P_{1, Q(1)} \mathbf{h}_{1, Q(1)}^{*} \mathbf{K}_{z_{1}}^{-1} \mathbf{h}_{1, Q(1)}
$$

and the corresponding ergodic capacity is given by

$$
\begin{aligned}
E\left\{C_{1}\right\} & =E\left\{\log _{2}\left[1+\gamma_{1}\right]\right\} \\
& =E\left\{\log _{2}\left[1+\lambda_{1, Q(1)}^{(1)} P_{1, Q(1)} \mathbf{h}_{1, Q(1)}^{*} \mathbf{K}_{z_{1}}^{-1} \mathbf{h}_{1, Q(1)}\right]\right\} \\
& =E\left\{\log _{2}\left[1+\lambda_{1, Q(1)}^{(1)} P_{1, Q(1)} \tilde{\mathbf{h}}_{1, Q(1)}^{*} \mathbf{R}_{1, Q(1)}^{* / 2} \mathbf{K}_{z_{1}}^{-1} \mathbf{R}_{1, Q(1)}^{1 / 2} \tilde{\mathbf{h}}_{1, Q(1)}\right]\right\} .
\end{aligned}
$$


Applying Jensen's inequality, it can be shown that

$$
E\left\{C_{1}\right\} \leq \log _{2}\left[1+E\left\{\lambda_{1, Q(1)}^{(1)} P_{1, Q(1)} \tilde{\mathbf{h}}_{1, Q(1)}^{*} \mathbf{R}_{1, Q(1)}^{* / 2} \mathbf{K}_{z_{1}}^{-1} \mathbf{R}_{1, Q(1)}^{1 / 2} \tilde{\mathbf{h}}_{1, Q(1)}\right\}\right]
$$

Since $E\left\{\tilde{\mathbf{h}}^{*} \mathbf{A} \tilde{\mathbf{h}}\right\}=\operatorname{tr}(\mathbf{A})$ and $\operatorname{tr}(\mathbf{A B})=\operatorname{tr}(\mathbf{B A})$, (14) can be rewritten as

$$
E\left\{C_{1}\right\} \leq \log _{2}\left[1+\operatorname{tr}\left(\lambda_{1, Q(1)}^{(1)} P_{1, Q(1)} \mathbf{K}_{z_{1}}^{-1} \mathbf{R}_{1, Q(1)}\right)\right]
$$

Similarly, it can be shown that the ergodic capacity of BS $i, i=1,2, \cdots, B$, is upper-bounded as

$$
E\left\{C_{i}\right\} \leq \log _{2}\left[1+\operatorname{tr}\left(\lambda_{i, Q(i)}^{(1)} P_{i, Q(i)} \mathbf{K}_{z_{i}}^{-1} \mathbf{R}_{i, Q(i)}\right)\right] .
$$

Thus, the ergodic capacity of the uplink MIMO cellular system is upper-bounded as

$$
E\left\{C_{\text {Total }}\right\} \leq \sum_{i=1}^{B} E\left\{C_{i}\right\} .
$$

It can be seen from (16) and (17) that the ergodic capacity of the uplink near the cell boundary in a MIMO cellular system is associated with the spatial correlation matrix of users. Provided that the BSs can share the spatial correlation matrix of users near the cell boundary, they can determine a group of users (e.g., one for each cell) that yields the maximum ergodic capacity. The procedure of the proposed cooperative user scheduling can be summarized as follows.

1. Estimate the spatial correlation of users near the cell boundary and share it with the adjacent BSs.

2. Estimate the ergodic capacity for all possible choices of user groups in (17). Then, determine the optimum target user $Q(i)$ and the corresponding time slot according to the scheduling policy (e.g., maximizing the total ergodic capacity $\left.E\left\{C_{\text {Total }}\right\}\right)$. Since all the BSs share the correlation information and have a common value $E\left\{C_{\text {Total }}\right\}$ in (17), they know which users are selected in the adjacent cells. Repeat this step until all users are group-wise selected.

3. Schedule users according to the scheduling policy. To maximize the output SINR, generate a receive weight in (11).

\section{Performance Evaluation}

The performance of the proposed multi-cell user scheduling scheme is verified by computer simulation. Since it is of concern on users near the cell boundary, the performance is evaluated in low SNR region. Common simulation parameters are summarized in Table 1. For comparison, the performance of the conventional MaxSNR with full instant CSI [5] and random scheduling is also evaluated.

Fig. 2 depicts the performance of the proposed scheme according to the number of users in a $(1 \times 4)$ highly
TABLE I

COMMON SIMULATION PRAMETERS

\begin{tabular}{|l|l|}
\hline \multicolumn{1}{|c|}{ Parameters } & \multicolumn{1}{c|}{ Setting } \\
\hline Cell configuration & Three sectored 19 cells (two-tier) \\
\hline Cell radius & $1 \mathrm{~km}$ \\
\hline Path loss & 4 \\
\hline Antenna configurations & $\begin{array}{l}1 \mathrm{Tx} \text { at each user, 4 Rx at the BSs } \\
\text { (SIMO) } \\
2 \mathrm{Tx} \text { at each user, 4 Rx at the BSs } \\
\text { (MIMO) }\end{array}$ \\
\hline Center frequency & 2.3 Ghz \\
\hline Channel & Raleigh fading \\
\hline Link adaptation & Ideal (Shannon capacity) \\
\hline
\end{tabular}

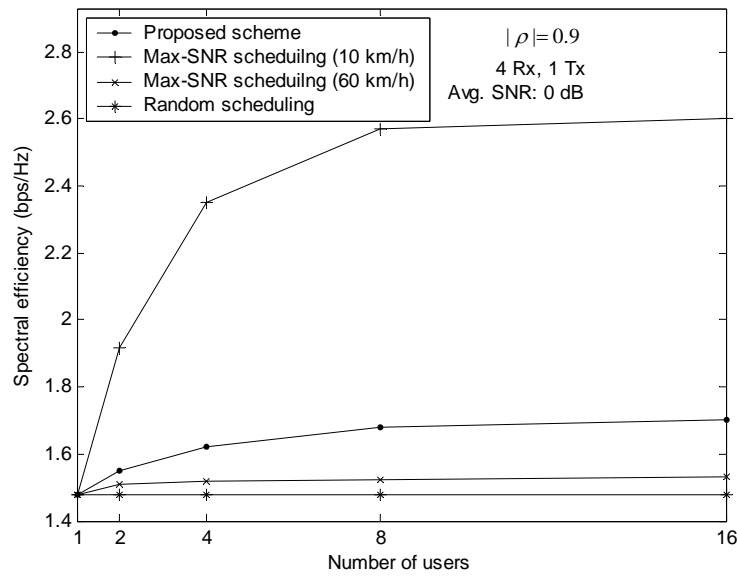

Fig. 2. Performance of the proposed scheme according to the number of users

correlated SIMO channel when users are uniformly distributed. The performance was estimated in two difference environments where user mobility is 10 and 60 $\mathrm{km} / \mathrm{h}$ due to mobility sensitivity of the Max-SNR scheduling. It is assumed that it takes $5 \mathrm{~ms}^{\text {delay }}{ }^{1}$ for the Max-SNR scheduling to start the transmission to the selected user. The actual channel of user $k$ can be represented using Jake's model as [14]

$$
\mathbf{h}_{i, k}^{\text {actual }}=\varepsilon \mathbf{h}_{i, k}^{\text {measured }}+\sqrt{1-\varepsilon^{2}} \hat{\mathbf{h}}_{i, k}
$$

where $\quad \hat{\mathbf{h}}_{i, k} \triangleq \mathbf{R}_{i, k}^{1 / 2} \tilde{\mathbf{h}}_{i, k} \quad$ is a random vector whose covariance is $\mathbf{R}_{i, k}$, and $\varepsilon$ denotes the time correlation coefficient between correlated channels (i.e., $\mathbf{h}_{i, k}^{\text {actual }}$ and $\mathbf{h}_{i, k}^{\text {measured }}$ ) given by [15]

$$
\varepsilon=J_{0}\left(2 \pi f_{d} \tau\right)+J_{2}\left(2 \pi f_{d} \tau\right)
$$

Here, $J_{k}(\cdot)$ the $k$-th order Bessel function of the first kind, $f_{d}$ is the maximum Doppler frequency, and $\tau$ represents the time delay between the instant of channel measurement and actual transmission [14].

It can be seen that the Max-SNR scheduling provides high performance gain when the user mobility is low. This is due to the fact that the Max-SNR scheduling can achieve

\footnotetext{
${ }^{1}$ It is frame interval in mobile WiMax system.
} 


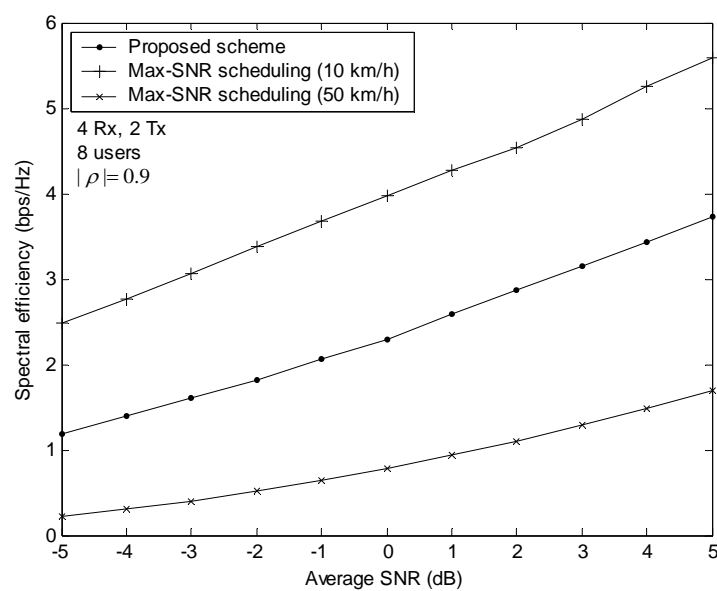

Fig. 3. Performance of the proposed scheme in MIMO environment according to the average SNR

instant channel gain (i.e., instant multi-user diversity gain) in low mobility environment as the number of users is increased. However, due to the channel mismatch problem, the Max-SNR scheduling degrades performance when the user mobility is high. On the other hand, the proposed scheme is quite insensitive to the user mobility as much as the random scheduling that utilizes no CSI. This is mainly because the spatial correlation is mobility invariant information, making the proposed scheme quite effective for mobile environments. It can be also seen that the proposed scheme provide high performance gain as the number of users is increased. This is mainly because as the number of users is increased, it is quite probable that users maximizing the ergodic capacity are grouped (i.e., the proposed scheme can achieve long-term multi-user diversity gain).

Fig. 3 depicts performance of the proposed scheme in a (2x4) highly correlated MIMO channel as a function of the average SNR when eight users are uniformly distributed in each cell at a mobility of 10 and $50 \mathrm{~km} / \mathrm{h}$. The transmit signal is weighted by the spatial correlation-based EVD and instant CSI-based MU-SVD [12] techniques in the proposed and Max-SNR schemes, respectively. It can be seen from Fig. 3 that the use of instant CSI is very susceptible to the user mobility. As a consequence, the Max-SNR scheduling outperforms when the the user mobility is low. It can also be seen that the proposed cooperative scheduling provides affordable performance regardless of the user mobility since it utilize time invariant channel information (i.e., spatial correlation information). Therefore, the proposed scheme can outperform the Max-SNR scheduling in high mobility environment.

\section{Conclusions}

We have considered cooperative user scheduling based on the spatial correlation information in the uplink of multi-cell mobile environments. Based on the spatial correlation information of users, the proposed scheme can improve the ergodic capacity by cooperatively scheduling users near the cell boundary to minimize the OCI. The proposed scheme is applicable to both the SIMO and MIMO environments, enabling the use of flexible mobile antenna schemes. The ergodic capacity of the proposed scheme has been analyzed using an upper bound and then verified by computer simulation. The simulation results show that the proposed scheme provides noticeable performance improvement over the conventional user scheduling schemes in highly correlated channel environments and that it provides performance less susceptible to the user mobility, working better than the Max-SNR scheduling scheme even in moderate mobility environments. In conclusion, the proposed scheduling scheme is quite effective in the presence of high channel correlation and/or user mobility.

\section{References}

[1] Z. Wang and R. Stirling-Gallacher, "Frequency reuse scheme for cellular OFDM systems,” Electron. Lett., vol. 38, no. 8, pp. 387-388, Apr. 2002.

[2] A. B. Carleial, "A case where interference does not reduce capacity," IEEE Trans. Inform. Theory, vol. 21, no. 5, pp. 569-570, Sept. 1975.

[3] K. W. Lee and Y. H. Lee, "Downlink beamforming for other-cell interference mitigation in correlated MISO channels,” in Proc. Eur. Wireless Commun.’07, Apr. 2007.

[4] 3GPP TR 25.814, Physical layer aspects for evolved UTRA, section 71.2.6, V7.1.0, Sept. 2006.

[5] S. Serbetli and A. Yener, "Time-slotted Multiuser MIMO systems: Beamforming and Scheduling Strategies,” EURASIP Jour. on Wire. Comm. Net., vol 2, pp. 286-296, 2004.

[6] M. Nicoli, O. Simeone and U. Spagnolini, "Multislot estimation of fast varying space-time communication channels," IEEE Trans. Signal Process., vol. 52, no. 5, pp. 1184-1195, May 2003.

[7] T. Salzer and D. Mottier, "On spatial covariance matrices for downlink eigen-beamforming in multi-carrier CDMA," in Proc. ICASSP'05, vol. 3, pp. 1133-1136, Mar. 2005.

[8] S. Zhou and G. B. Giannakis, "Optimal transmitter eigenbeamforming and space-time block coding based on channel correlations,” IEEE Trans. Inform. Theory, vol. 49, no.7, pp.16731690, July 2003.

[9] A. Paulaj, R. Nabar, and D. Gore, Introduction to Space-Time Wireless Communications, Cambridge, 2003.

[10]P. J. Smith, S. Roy, and M. Shafi, "Capacity of MIMO systems with semicorrelated flat fading,” IEEE Trans. Inform. Theory, vol.49, no. 10, pp.2781-2788, Oct. 2003.

[11]D. Tse and P. Viswanath, Fundamental of Wireless Communication, Cambridge, 2005.

[12]F. Jiang, Y. Wang, G. Liu, X. Fang and P. Zhang, "Exploiting multiuser spatial diversity in MIMO OFDM system through uplink scheduling,” in Proc. IEEE WCNC’07, pp. 953-957, Mar. 2007.

[13]A. Narula, M. Lopez, M. Trott, and G. Wornell, "Efficient use of side information in multiple-antenna data transmission over fading channels,” IEEE J. Select. Areas Commun., vol. 16, pp. 1423-1436, Oct. 1998.

[14]J. G. Proakis, Digital Communications, 4th ed., McGraw-Hill, 2001.

[15]G. D. Durgin and T. S. Rappaport, "Theory of multipath shape factors for small-scale fading wireless channels," IEEE Trans. Antennas Propag., vol. 48, no. 5, pp. 682 - 693, May 2000. 